$$
\text { "Engbersen" — 2009/11/2 — 0:13 — page } 297 \text { — \#1 }
$$

\title{
Comparison of teaching exponential and logarithmic functions based on mathematics textbook analysis
}

\author{
ArANKa ENGBERSEN
}

Abstract. Exponential and logarithmic functions are key mathematical concepts that play central roles in advanced mathematics. Unfortunately these are also concepts that give students serious difficulties. In this paper I would like to give an overview - based on textbook analysis - about the Hungarian, Austrian and Dutch situation of teaching exponential and logarithmic functions. This comparison could also provide some ideas for Hungarian teachers on how to embed this topic in their practice in another more "realistic" way.

Key words and phrases: exponential and logarithmic functions, realistic mathematics education, horizontal vs. vertical mathematization, concept image.

ZDM Subject Classification: C30, D40, I20.

\section{Introduction}

One of the most important topics in secondary mathematics teaching is the exponential and logarithmic functions both from mathematical and applications point of view. It belongs to the curriculum for grades 10 and 11 in most MiddleEuropean countries. At this level in Hungary the so called "top down" style of mathematics teaching is typical what is stressing much of mathematics based on deductive proof, not on exploration and experimentation. Mathematics educators agree that we can influence the mathematics teaching practice mainly by two factors: by mathematics textbooks and by mathematics exams. In the present article we compare three mathematics textbooks, one from Austria, one from

Copyright (c) 2009 by University of Debrecen 


$$
\text { "Engbersen" — 2009/11/2 — 0:13 — page 298 — \#2 }
$$

Hungary and finally one from the Netherlands. We have chosen the last one consciously, because it mirrors the ideas of the realistic mathematics education reform movement. In all three cases we will analyse the topics of exponential and logarithmic functions. The authors of Hungarian mathematics textbook are practicing secondary school teachers, so the textbook really reflects the typical Hungarian traditions concerning the mathematics teaching.

Our opinion is that it is valuable to know different teaching cultures, to try to profit from them and to avoid a narrow point of reference in relations to views, beliefs and attitudes.

\section{Some theoretical remarks Concept Rich Mathematics Instruction}

Our theoretical analysis will be based on the ideas of the "Concept-Rich Mathematical Instruction". (Meir Ben-Hur, 2005)

It has two basic principles: learning new concepts reflects a cognitive process and secondly this process involves reflective thinking that is greatly facilitated through mediated learning. This teaching method has five components: Practice - De-contextualization - Meaning: Encapsulating a generalization in words Re-contextualization - Realization.

\section{Practice}

Learning direct instruction practice is considered as drill that is followed by the presentation and demonstration of the concept. (Showing examples, checking for understanding, guided practice that drills students on the use of the concept). The Concept-Rich approach involves novel activities that are all based on the same concept. The teacher starts with an intriguing problem and later provides a variety of other problems, so the students experience different ways of conceptual applications so that the concepts they draw out from their experiences grow beyond the incidental properties of the problems. Piaget (1995a) considered conceptualization as a shift of operation from the plane of action, in which misconceptions or other difficulties interfere and create confusion, to the plane of thought. He stated there is a new and unique experience, once it is over it is enacted upon in thought on the verbal plane.

Students may say the right thing, but they may fail to enact it. They may do the right thing, but it is possible that they cannot explain it. Students lose 
"Engbersen" — 2009/11/2 — 0:13 — page 299 — \#3

premature concepts over time if they do not continue to practice and reflect upon them. The conceptualization depends on the amount - the nature - and the novelty and challenge of the practice structured by the teacher. Research shows that for some students practice without guidance does not necessary solve the problems created by shortened teacher-guided practice. The low achieving students need more time and mediation to form the concepts. Learning has a nonlinear nature new experiences require more time and effort than later experiences. The early experiences must be simple, and only the later ones must be progressively more complex. Students must experience new exercises connected to the concept repeatedly until they have crystallized the concept in their mind.

\section{De-contextualization}

This component has a decisive role in effective concept building. Experts generally agree that concepts cannot simply be passed from one person to another by talk, because each individual must abstract concepts from his or her own experience. Piaget (1995b) showed that the process starts with analogies among different individual cases, which gradually lead to an extrapolation of a common principle that can be extended over the whole universe of experience. Students gradually learn to consider, analyse and compare the procedure without a need to perform it, or understand the conditions under which it works. Students learn to see mathematical processes in their entirety. This reflective process is facilitated by language (writing or classroom discourse). Effective teachers determine the quality of de-contextualization. Variable and sufficient practices help students to recognize the relationships existing among the different representations of the concept and identify their core similarities. The classroom discours develop the students' understanding. The teacher can enhance the de-contextualization process using "higher-order" questioning. These questions involve comparing experiences, grouping them, generalizing common ideas from such comparisons, anticipating future experiences based on generalizations. Such questions may not be raised in the learners' minds without the teachers' mediation. There are two types of higher order questioning: analysing questions - call for arguments to support or reject given positions, call for different views, solutions, examine errors; inference questions - facilitate the process if "filling in" missing information.

Another factor is the wait time. Research showed that low-achieving students do not have enough time in the lessons to reflect and respond to teachers' questions. Teachers should wait longer and listen carefully to students' responses and asking probing questions when it is necessary.
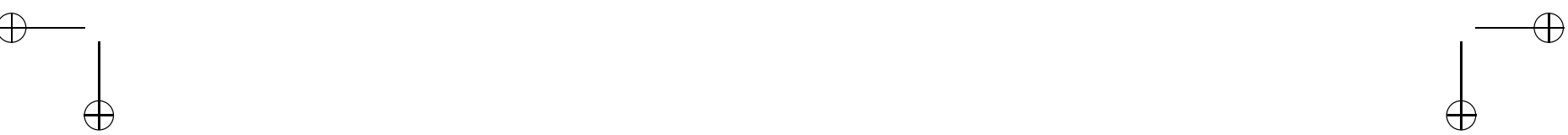


$$
\text { "Engbersen" — 2009/11/2 — 0:13 — page 300 — \#4 }
$$

Students shall recognize that many strategies may work in solving of a problem. It is an important task of the teachers to encourage divergent responses.

\section{Meaning: Encapsulating a generalization in words}

Students must learn to define concepts and elaborate upon them in their general form. They must learn to encapsulate their conceptual understanding in words and symbols, so they get new meaning. This cognitive process is derived from a multitude of experiences. It is a complex enterprise and requires careful teacher mediation to compare and de-contextualizate concepts gained within this process. It involves the implementation of the new concept in a hierarchy of known concepts. Meaning should guide the thinking.

Some teachers understand that learning concepts mean to explain them to the students. Others have the opinion that students independently construct concepts from their own experiences. Education research supports neither of these views. First of all, discovery learning does not work in case of lower achievers. Discovery alone certainly cannot produce the whole logical structure of the concept for students; they need guidance in forming new mathematical concepts.

\section{Re-contextualization}

Experts argue that conceptualization is complete only if the learner is able to re-contextualize the concept in a new and untried experience. New concepts do not get automatically transferred, it must be coached. The students' mind needs to clear old connections based on misconceptions or on irrelevant features. Teachers need to give time and direct help to engage students in the application process.

\section{Realization}

The test of learning is the realization of what has been learnt and is applicable across the curriculum and in everyday life.

Realization means that students' classroom experiences are meaningful and useful activities. In the mathematics lessons there should be maintained an atmosphere of fostering the students' realization of new concepts. In this article we do not deal with concrete classroom realization. 


$$
\text { "Engbersen" — 2009/11/2 — 0:13 — page } 301 \text { — \#5 }
$$

\section{Concept image}

David Tall and Shlomo Vinner introduced this notion into the international mathematics didactics scientific literature. We call a mental object concept image of a concept associated with the full cognitive structure, which contains the visual representations (pictures, diagrams, graphs), mental representations (connections), concrete experiences, examples, properties, procedures, delightful experiences, stories in connection with the concept. (D. Tall, S. Vinner 1981)

From their research it is quite clear that a formal definition of a concept does not lead necessarily to an effective acquisition of it, a rich concept image plays a dominant role too.

To relate the concept image to Concept Rich Mathematics Instruction, we may state that it should be one of the main aims of mathematics instruction to help students to build a rich concept image to more and more concepts. The practice and de-contextualization phases may ensure such construction processes.

\section{Instrumental and relational understanding}

According to Richard Skemp "to understand" something means to assimilate it into a person's appropriate schema the person has.

Instrumental understanding means knowing "how" without "why".

Relational understanding means knowing both "how" and "why".

There is the danger when teaching a topic that the students can use mechanically the relevant rules without having a conscious knowledge about them. (R. Skemp, 1987)

\section{Realistic Mathematics Education}

The frounder of this reform movement is Freudenthal. His main view was that mathematics should never be presented to the students as a ready-made product. The opposite of this view is seeing mathematics in statu nascendi. He considered mathematics as a human activity. He criticized strongly the modernist approach because of its antididactical characteristic. (Freudenthal, 1973) Mathematics instruction should start not with the formal system, but with the final product. The phenomena by which concepts appear in reality should be the 


$$
\text { "Engbersen" — 2009/11/2 — 0:13 — page 302 — \#6 }
$$

source of concept formation. According to Treffers the starting points of realistic mathematics education are:

- Paying much attention to "re-invention", recreating mathematical concepts and structures on the basis of intuitive notions in the making of made (active)

- Carrying on at various levels of concreteness and abstraction (differentiated)

- The programming of instruction is guided by historical-genetic rather than subject matter systematic method (vertically planned)

- Reality bound meaningful (mathematically rich) instruction. (Treffers, 1986)

The basic idea of realistic mathematics education is to start from a concrete situation, to perceive the essentials, to formulate them in mathematical terms and to construct an abstract model of the initial situation.

The mathematization process has two components.

Horizontal mathematization

It means transferring the problem to a mathematically stated problem. With help of visualization and schematization The students try to discover regularities and relations, for which is necessary to identify the specific mathematics in a general context.

Activities are:

- discovering relations;

- discovering regularities;

- recognizing isomorphic aspects in different problems;

- transferring a real world problem to a mathematical problem;

- transferring a real world problem to a known mathematical model.

Vertical mathematization

As the problem has been transferred to a - more or less - mathematical problem, this problem can be attacked with mathematical tools.

Activities:

- representing a relation by a formula;

- proving regularities;

- refining and adjusting models;

- using different models;

- combining and integrating models;

- formulating a new mathematical concept;

- generalizing. 


$$
\text { "Engbersen" — 2009/11/2 — 0:13 — page 303 — \#7 }
$$

Comparing the Concept Rich Mathematic Instruction ideas with the Realistic Mathematics Education we may notice that the Practice, De-contextualization, Re-contextualization (transferring a real world problem to a mathematical problem and the knowledge of a mathematical model) parts are contained in horizontal mathematization, the vertical mathematization is not presented in details in the first one.

\section{Teaching-suggestions for exponential and logarithmic functions in different textbooks}

The concepts of this topic are close to each other: extending of the powers for rational, irrational exponents, identities of the powers, exponential functions, exponential equations, inequalities, logarithm, identities for the logarithms, logarithmic functions, logarithmic equations, inequalities, system of equations. It is not easy to choose one of them to characterize that its teaching is not taking into consideration the teaching of other notions. For this reason too we investigate these concepts in their units placing emphasis on the teaching of exponential and logarithmic functions.

\section{Colourful Mathematics 11 (Hungary)}

The main title of the chapter shows clearly the statement above: "Power, root, logarithm". At the beginning of the chapter there is a summary of the earlier learnt material: identities of powers and roots, emphasizing the analogy between them. (Power of the product $-n$-th root of the product) Some power and root functions, their graphs. (Applying transformations)

\section{Powers with rational exponents}

There is no "practice", no "de-contextualization" part, the authors start with "Encapsulating a generalization with words". "We want to extend the concept of the power for all rational exponents, preserving the validity of the identities for integer exponents."

$\left(7^{\frac{2}{3}}\right)^{3}=7^{2}$ Based on the identity of power of power

$\left(\sqrt[3]{7^{2}}\right)^{3}=7^{2}$ Based on the definition of the third root

Let be $7^{\frac{2}{3}}=\sqrt[3]{7^{2}}$. 


$$
\text { "Engbersen" — 2009/11/2 — 0:13 — page } 304 \text { — \#8 }
$$

Definition. The $m / n$-th power of the positive number $a$ is the $n$-th root of the $m$-th power of $a$.

$a^{\frac{m}{n}}=\sqrt[n]{a^{m}}$ where $a>0 ; m \in \mathbb{Z}, n \geq 2$ and $n \in \mathbb{N}$.

There is a remark why " $a$ " cannot be negative resp. 0 .

It follows a theorem with its proof: If $a>0 ; k, l, m, n \in \mathbb{Z} ; n, l \geq 2$ and $\frac{m}{n}=\frac{k}{l}$ than $a^{\frac{m}{n}}=a^{\frac{k}{l}}$.

There are a lot of exercises for applying the identities for rational exponents.

The introduction of power for irrational exponents is a hard task for secondary school. There is an example in the textbook, how we can approximate the value of $2^{\sqrt{5}}$ with help of intervals, based on the monotone property of the function: $2^{x}$.

Definition. We call the function $f: \mathbb{R} \rightarrow \mathbb{R} ; f(x)=a^{x} ; a>0$ exponential function.

It follows the graphs of exponential functions for $a<0<1 ; a=1$; and $a>1$ and some transformations of the functions $f(x)=2^{x}$ resp. $g(x)=\left(\frac{1}{2}\right)^{x}$.

Between the solved tasks there is only one application problem (change of the pressure of air depending on the height) and between the exercises there is only one application (radioactive decay). There are a large amount of exponential equations, inequalities, system of equations. Most of them can be solved with help of identities, but some of them are quite complex, requiring unusual concepts and solutions due to Hungarian traditions.

\section{The concept of logarithm}

There is a realistic task for introduction: In a laboratory the experts are counting the bacteria. The number of bacteria doubles in every hour. How much time is needed to reach 10000 bacteria, necessary for one experiment, if at the beginning there are 800 bacteria?

The presented solution leads to the equation: $2^{x}=12,5$ Based on the monotony of the function, the textbook gives a result with a hundredth accuracy. After this task follows the definition of the logarithm.

Definition. The logarithm of the number $b$ with base $a$ is the exponent, powering $a$ for it we get the number $b$, where $a<0 ; a \neq 1$ and $b>0$. Notation: $\log _{a} b$. 


$$
\text { "Engbersen" — 2009/11/2 — 0:13 — page 305 — \#9 }
$$

Among the solved and unsolved problems (exercises) there are some for determining the value of the logarithmic expressions and some for finding the domain of them. There is only one task concerning radioactive decay.

The logarithmic function is introduced as the inverse of the relevant exponential function. It is followed by some tasks for drawing the graphs of logarithmic functions (with help of transformations too). Here we find one realistic task about ornamental plants.

The identities for the logarithm of products, fractions and powers are followed by their proofs. In the last part there are numerous logarithmic equations, inequalities, systems of equations, some from them really demanding.

It is interesting to mention that there is only a small part in the textbook for practical applications: four realistic problems occur with their solution. (cooling down of a liquid, loss of value of a machine, radioactive decay, drug consumption)

\section{Summary}

The Hungarian elaboration of our topic is an example for "top down" presentation style which is represented very often as a top down teaching style in the classroom. Mathematics as a science stands in the centre. The "decontextualization" phase is very short, and at some concepts it fails. At many concepts the authors start with the "Encapsulating the Generalization in Words" on a quite abstract level. It seems that for many-many students these concepts are very difficult. For these students the re-contextualization is a very hard task because the lack or the failure of de-contextualization.

\section{Kronfellner-Peschek: Angewandte Mathematik (Austria)}

\section{Exponential functions}

The authors start with an explanation: "For many no mathematical processes yield, that the relative (percent) increase and decay of the process is approximately constant. We call the mathematical model of these processes as exponential functions."

Problem 1. One Biology researcher observes that the area of a cell culture increases in every hour by $45 \%$. Find the area after $1,2,3,4,5, n$ hours, if the area was $1000 \mathrm{~mm}^{2}$ at the beginning. 


$$
\text { "Engbersen" — 2009/11/2 — 0:13 — page 306 — \#10 }
$$

SOLUTION.

$$
\begin{aligned}
& A(1)=1000+1000 \cdot 0,45=1000 \cdot 1,45 \approx 1450 \\
& A(2)=A(1)+A(1) \cdot 0,45=1000 \cdot 1,45^{2} \approx 2100 \\
& A(3)=A(2) \cdot 1,45=1000 \cdot 1,45^{3} \approx 3000 \\
& A(4)=A(3) \cdot 1,45=1000 \cdot 1,45^{4} \approx 4400 \\
& A(5)=A(4) \cdot 1,45=1000 \cdot 145^{5} \\
& A(n)=1000 \cdot 1,45^{n}
\end{aligned}
$$

This solutions shows that the assumption - $45 \%$ increase in every hour - leads to the formula while the relative increase per hour is constant.

$$
A(n+1)=A(0) \cdot 1,45^{n+1}=A(0) \cdot 1,45^{n} \cdot 1,45=A(n) \cdot 1,45
$$

It seems plausible to assume that not only in every hour is the increase the same (from where do the cells "know" that one hour is over?). We will show that in every half, quarter and generally in every time interval with equal length the relative increase is equal. But to do so it we must assume that the formula $A(n)=A(0) \cdot 1,45^{n}$ is valid for non integers $n$ too.

Problem 2. a. Find the increase of the area after the first half of an hour.

b. Show that in the 2., 3., 10. half of an hour the area increases by the same rate.

c. Show that in every half of an hour the increase rate is the same.

Solution of $\mathrm{C}$. We choose an arbitrary time point $t$ and calculate the relative increase in the time interval $[t ; t+05]$

$$
\begin{aligned}
A(t) & =1000 \cdot 1,45^{t} \\
A(t+0,5)=1000 \cdot 1,45^{t+0,5} & =1000 \cdot 1,45^{t} \cdot 1,45^{0,5}=A(t) \cdot 1,45^{0,5}
\end{aligned}
$$

Problem 3. a. How many percent does the area increase by in the first quarter of an hour?

b. Show that the area changes in every quarter of an hour by the same increase rate. 
Problem 4. Show that in every equal length time-interval the area increases by a rate that depends only on the lengths of the interval.

Let us consider the time interval $[t ; t+h]$. We can show that $A(t+h)=$ $A(t) \cdot 1,45^{h}$ holds. That means that the area increases by the change rate $1,45^{h}$. This factor depends only on $h$, but not on $t$.

We can find in nature and in the technical processes cases where it is plausible to assume a constant relative change per time unit is and this assumption can be justified (in good approximation) by observation (experiments). We can characterize these processes by the formula $A(t)=c \cdot a^{t}$ or $A(t+1)=A(t) \cdot a$.

Definition. A real function $f$ with $f(x)=c \cdot a^{x}\left(c \in \mathbb{R} ; a \in \mathbb{R}^{+}\right)$is called exponential function and we call the equations $f(x)=c \cdot a^{x}$ resp. $y=c \cdot a^{x}$ exponential equations.

Problem 5. The number of the bacteria in a culture will be approximately described by the formula $N(t)=200 \cdot 1,7^{t}$ ( $t$ in hours)

a. Find the number of bacteria after 1, 2, 5, 10 hours.

b. Show that the number of bacteria increases in every hour, in every half a hour, in every quarter of an hour by the same change rate.

Problem 6. Show in general that every exponential function $f$ with $f(x)=$ $c \cdot a^{x}$ has the following properties has: $f(x+1)=f(x) \cdot a$ resp. $f(x+h)=f(x) \cdot a^{h}$.

Further tasks for exponential processes (as problems task to be solved)

- radioactive decay (4 tasks)

- bacteria

- air pressure depending on height

- sugar melting

- brightness of the light in a liquid

- population growth (2 tasks)

- germs in cow milk

Properties of the exponential functions:

- The values of the exponential functions are positive

$-f(x)=a^{x}$ for $a>1$ is increasing, for $0<a<1 f(x)$ is decreasing 


$$
\text { "Engbersen" — 2009/11/2 — 0:13 — page 308 — \#12 }
$$

Comparison of the linear and the exponential growth

Linear function: $f(x)=k x+d$

$$
f(x+h)-f(x)=k h
$$

Exponential function: $f(x)=c \cdot a^{x}$

$$
\frac{f(x+h)}{f(x)}=a^{h}
$$

These properties are visualized by graphs.

\section{Logarithm}

Introductory problem:

a. There are 25 water lilies in a lake. We assume that the number of lilies doubles in every day. How many days are needed to have 400 water lilies?

b. A radioactive material decomposes by the formula $N(t)=N_{0} \cdot 0,8^{t}$ ( $t$ in years). How much time is needed to reach the $10 \%$ remained material?

The solution of the first problem leads to the equation $2^{t}=16$. The solutions is $t=4$.

At the second problem the substitution leads to the equation: $0,8^{t}=0,1$. It follows a trial with help of approximation using rational exponents. The results are summarized in a table and it ends with the following interval: $t \in[10,3 ; 10,35]$.

Using the graphs of relevant exponential functions to find the exponent with help of drawing, follow 9 problems.

The authors emphasize that the common task was to find the value of the exponents at the equations form $a^{x}=y\left(a, y \in \mathbb{R}^{+}\right)$. These exponents are called logarithm of $y$ to the basis a.

Definition. Let $a, y \in \mathbb{R}^{+}, a \neq 1$. The number $x \in \mathbb{R}$, for it yields $a^{x}=y$ is called the logarithm of $y$ to the basis $a$. Symbolic: $a^{x}=y \Longleftrightarrow x=\log _{a} y$. In words: The logarithm of $y$ to the basis $a$ is the number, powering $a$ with it we get $y$.

There follow numerous problems to find the value of logarithmic expressions. From our point of view as re-contextualization follow 8 word problems, where we have to apply the logarithm to come to the solution.

- Bacteria 


$$
\text { "Engbersen" — 2009/11/2 — 0:13 — page 309 — \#13 }
$$

- Cow milk

- Increase of the economy

- Population growth

- Brightness of the light in the liquids

- Radioactive decay (2 tasks)

- Growth of salaries

Finally the identities of logarithm and their proofs are presented and some tasks for applying these rules. The chapter ends with the logarithm to the base $e$ (natural logarithm).

\section{Summary}

The concept of the exponential growth will be practiced in one context, investigating different time intervals. This might help most students to understand and to practice the exponential growth. For de-contextualization there are enough problems. We can state that the phases - practice, de-contextualization, encapsulating the generalization in words, re-contextualization - at both concepts (exponential growth, logarithm) means a good didactical model. The problems are real life problems. The main task is not to solve complicate equations, but to find the right mathematical models. This style of helping teaching may be considered as "bottom-up" version.

The Dutch version: realistic mathematics instruction

Introduction, exponential growth and decay

Problem 1. On their $9^{\text {th }}$ birthday two friends Anna and John both get their first pocket money. Both of them get 50 EUR for every month until their next birthday. Anna is promised to receive by every coming birthday 10 EUR more for each month and John is said to increase his money with $20 \%$ for every month by each following birthday.

a. How much money will they get monthly after their $11^{\text {th }}$ birthday?

b. Complete the following table, but first explain what has already been written: 


\begin{tabular}{|l|l|l|}
\hline birthdays & Anna's money amount & John's money amount \\
\hline $9^{\text {th }}$ & 50 & 50 \\
\hline $10^{\text {th }}$ & $50+10=60$ & $50+0.20 \times 50=60$ \\
\hline $11^{\text {th }}$ & $60+10=$ & $60+0.20 \times 60=$ \\
\hline $12^{\text {th }}$ & & \\
\hline $13^{\text {th }}$ & & \\
\hline $14^{\text {th }}$ & & \\
\hline
\end{tabular}

The pocket money of both children can also be graphed.

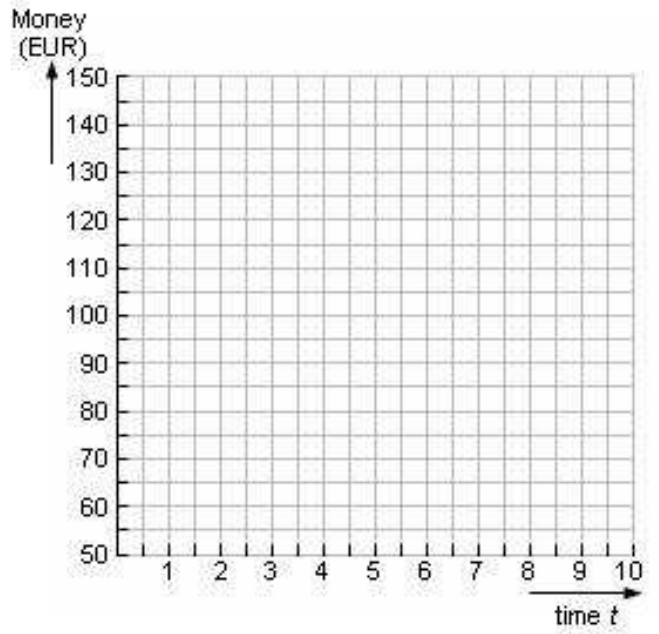

(in years)

c. Use the grid to plot the points you found in the table.

d. Do you think either of these growth patterns is realistic for a longer period of time?

e. What is the most striking difference between the two graphs?

The kind of growth of Anna's money is called linear growth. Every year a fixed number (10) is added to the amount of the previous year. We may call Anna's money $M_{A}$ and the time after the $9^{\text {th }}$ birthday date $t$ (in years).

f. What function describes the relationship between $M_{A}$ and $t$ ? What type of function is it?

The growth of John's money deserves a closer look! Looking at the amounts in the table, you can find the following relationship:

amount/month this year : amount/month last year $=1.2$
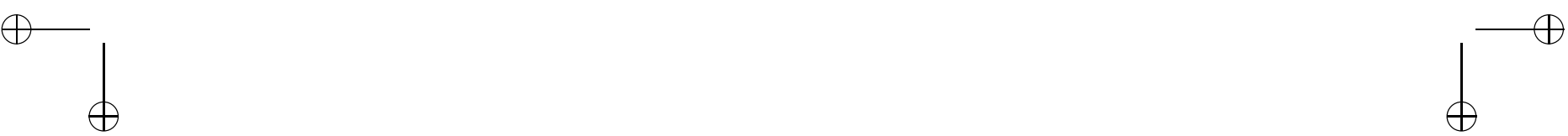
g. Check this ratio for each pair of successive year and explain why this is correct for this kind of growth.

h. Explain why the function $M_{J}(t)=50 \cdot 1.2^{t}$ describes the relationship between $M_{J}(t)$ and $t$ !

A growth process that can be described with a function like

$$
G(t)=c \cdot g^{t}
$$

is called an exponential growth process.

In this function, $c$ is often called the initial value and $g$ (the base) the growth factor.

The name "exponential" comes from the fact that the independent variable $(t)$ is an exponent. Mathematically spoken, the growth factor $g$ is called the base, but for the time being we will call it growth factor because that makes more sense in applications.

Although the growth of money amounts described in the two different ways is not very realistic for the long run, it showed some interesting differences between linear and exponential growth:

Linear growth is caharcterized by the following property: Every fixed time step results in a fixed additition.

Exponential growth is characterized by: every fixed time step results in a fixed multiplication.

Problem 2. The previous example of exponential growth was about increasing numbers. But exponential processes are also possible with decreasing numbers. The word 'growth' is a little strange for normally we talk about 'decay' when the numbers are decreasing.

An example of exponential decay is the half-life of medicines, alcohol, radioactive materials and caffeine. The half-life, or time it takes for the amount of caffeine in the blood to decrease by $50 \%$, ranges from 3.5 to 10 hours. In adults the half-life is generally around 5 hours. A 7 oz cup filled with Espresso has $100 \mathrm{mg}$ caffeine. I (an adult person) drink an Espresso at $11 \mathrm{am}$.

a. How many mg of caffeine are still in my body when I go to bed at $11 \mathrm{pm}$ ?

One day I drink an Espresso at 11am and another one at $5 \mathrm{pm}$.

b. How many mg of caffeine are still in my body now when I go to bed at $11 \mathrm{pm}$ ? 
c. What is the 'growth factor' of caffeine per hour for an adult?

Describe the relationship between the amount of caffeine $C$ (in $\mathrm{mg}$ ) in the body as a function of the time $t$ (in hours) after drinking a 7 oz cup of Espresso.

Real-life exercises in more mathematical aspect

Problem 1. Escherichia coli (E.coli) is one of the main species of bacteria that live in the lower intestines of mammals including us. They are necessary for the proper digestion of food and are part of the intestinal flora. E.coli is important for digestion. Incredible, but they double every two hours!

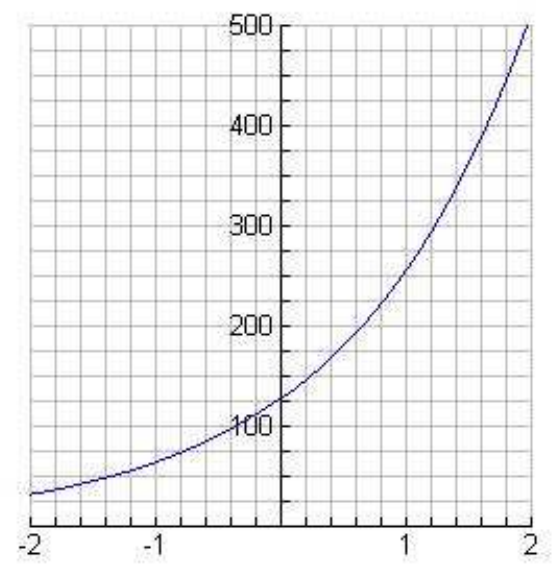

a. Draw the graphic of the growth with $t(1,2,3,4,5)$.

b. Let us look at that situation when at the moment of our first observation there are already 128 bacteria. Complete the following table:

\begin{tabular}{|c|c|c|c|c|c|c|c|c|c|}
\hline$t$ (time,hours) & -4 & -3 & -2 & -1 & 0 & 1 & 2 & 3 & 4 \\
\hline$N(t) /$ number of bacteria & & & & & 128 & & & & \\
\hline
\end{tabular}

d. What is the formula that describes this growth? $V(t)=128 \cdot 2^{t}$ with $t$ the time (in units of 2 hours) and $V$ the volume of E.coli. Make a graph of the growth!

e. Make an estimate of the volume of E.coli after one hour (this is at the moment $t=0.5$ !) and also after 3 hours $(t=1.5)$. 
f. How many were there at $t=-1$ and $t=-2$ ?

$$
\begin{aligned}
& 2^{-1}= \\
& 2^{-2}= \\
& 2^{-n}=
\end{aligned}
$$

What is going to happen if I need details about:

$$
\begin{aligned}
& 2^{1 / 2}= \\
& 2^{1 / 4}=
\end{aligned}
$$

g. Let us have a look at the graph of $g: \mathbb{Q} \rightarrow \mathbb{R} g(x)=2^{x}$ and $f(x)=3^{x}$. The two graphs pass the vertical axis at height $y=1$. Explain why this is true for all exponential functions $y=b^{x}$.

For $b>1$, the graph of $y=b^{x}$ gets closer to the horizontal axis as $x$ becomes more and more negative (or mathematically speaking: $x$ approaches $-\infty)$. But the graph will not cross the axis or even touch it.

We say that the (negative) $x$-axis is the horizontal asymptote of the function.

Exponential decay is comparable to exponential growth. A 'growth factor' of $\frac{1}{2}$ is the opposite of a growth factor of 2 : instead of doubling every time unit (growth factor $=2$ ), the amount is halved every time unit (growth factor $=\frac{1}{2}$ ). So, exponential functions with a base $(b)$ between 0 and 1 are similar to exponential functions in which the base $\mathrm{b}$ is greater than $1(b>1)$.

h. Below you see the graphs of $f(x)=3^{x}$ and $g(x)=\left(\frac{1}{3}\right)^{x}$ in one coordinate system.

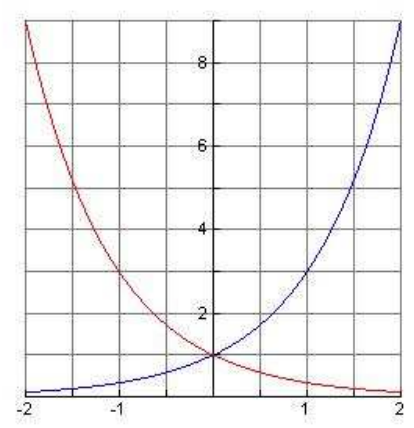


i. Describe what you notice.

j. Describe in words the meaning of $g(x)=f(-x)$

k. Use algebra to prove that $g(x)=f(-x)$ is true for the two given functions.

l. Consider the function $f(x)=3^{x}$ for all real numbers.

m. Is $f(x)=3^{x}$ interpretable if $x$ is an irrational number? For example how would you define $3^{\sqrt{5}}$ ?

The exponential function $f: \mathbb{R} \rightarrow \mathbb{R}$ with base $b$ is defined by
\[ f(x)=b^{x} \text { or } y=b^{x} \]
where $b$ is a positive constant other than 1 and $x$ any real number.

\section{Logarithm}

Problem 1. The sea brown alga is growing very fast. Every week it doubles its height. Beginning with $1 \mathrm{~m}$ of height of an alga on one day (starting time $t=0)$, after 1 week $(t=1)$ it will be $2 m$ high. The formula that describes this process is $h=2^{t}$.

a. Using a graph answer the following question: At what moment is the height $20 m$ ?

b. Now without the help of graph at what moment are will the alga 40, 80, 10, 5 $m$ high? The same question for $3 m$ (with the graph) and $6,12,36 m$ without graph.

c. Fill all empty cells in the following tables:

\begin{tabular}{|c|l|l|l|l|l|l|}
\hline $\begin{array}{c}\text { Height } \\
(m)\end{array}$ & 1 & 2 & 4 & 8 & 16 & 32 \\
\hline $\begin{array}{c}\text { Time } \\
\text { (weeks) }\end{array}$ & 0 & 1 & & & & \\
\hline
\end{tabular}

\begin{tabular}{|c|l|l|l|l|l|l|}
\hline $\begin{array}{c}\text { Height } \\
(m)\end{array}$ & 3 & 6 & 12 & 24 & & \\
\hline $\begin{array}{c}\text { Time } \\
\text { (weeks) }\end{array}$ & 1.6 & & & & & \\
\hline
\end{tabular}

\begin{tabular}{|c|l|l|l|l|l|l|}
\hline $\begin{array}{c}\text { Height } \\
(m)\end{array}$ & 2.5 & 5 & 10 & 20 & & \\
\hline $\begin{array}{c}\text { Time } \\
\text { (weeks) }\end{array}$ & & 2.3 & & & & \\
\hline
\end{tabular}

\begin{tabular}{|c|l|c|l|l|l|l|}
\hline $\begin{array}{c}\text { Height } \\
(m)\end{array}$ & & $\begin{array}{c}0.2 \\
5\end{array}$ & 0.5 & 1 & & \\
\hline $\begin{array}{c}\text { Time } \\
\text { (weeks })\end{array}$ & & & & 0 & & \\
\hline
\end{tabular}

d. Here we are looking at $t$ as a function of $h$, you can think of $t(3)$ as "the time it takes to grow from $1 \mathrm{~m}$ to $3 \mathrm{~m}$ ".

Earlier we have learnt the exponential function that related height to time:

$$
\text { Time } \underset{\text { function }}{\stackrel{\text { exponential }}{\longrightarrow}} \text { height }
$$


Recall that the characteristic of exponential growth (of an exponential function) is:

Exponential function: every fixed time step in a fixed
multiplication of the area
Exponential function: every fixed time step results in a fixed
multiplication of the height

Now we are exploring a function that operates the other way:

Time $\frac{\text { logarithmic }}{\text { function }}$ height

Because a logarithmic function is the inverse function of an exponential one, the characteristic of a logarithmic function that relates height to time is:

For this specific case of the sea brown alga the growth factor is 2 , which should be reflected in the notation of the logarithm. The notation for the logarithmic function that

Logarithmic function: every fixed multiplication of the area results in a fixed time

relates height $h$ to time $t$ is: $t=\log _{\text {growthfactor }} h$.

Since the growth factor is 2 , the logarithmic function should be $t=\log _{2} h$

$\log _{2} 10$ is the time needed to grown to ten

times its initial area (when the growth factor is 2)

e. Using the alga context try to explain the following expressions

$$
\begin{array}{llll}
\log _{2} 1=0 & \log _{2} 4=2 & \log _{2} 8=3 & \log _{2} 32=5 \\
\log _{2}\left(\frac{1}{2}\right)=-1 & \log _{2}\left(\frac{1}{4}\right)=-2 & \log _{2}\left(\frac{1}{16}\right)=-4 & \log _{2}\left(\frac{1}{64}\right)=-6 \\
\log _{3} 27=3 & \log _{3} 81=4 & &
\end{array}
$$

Explain the following equations:

$$
\begin{array}{ll}
\log _{2} 3+1=\log _{2} 6 & \log _{2} 6+1=\log _{2} 12 \\
\log _{2} 10+1=\log _{2} 20 & \log _{2} 5+1=\log _{2} ?
\end{array}
$$

If you add the number 1 to a logarithm (like $\log _{2} 3$ ), you should realize that $\log _{2} 3$ is a time period. So the number 1 in the equation also needs to be a time period. Therefore 1 can be replaced by $\log _{2} 2$, which is the time needed to double with a growth factor of 2 . This is exactly one week.

So: $\log _{2} 3+1=\log _{2} 6$ can be rewritten as $\log _{2} 3+\log _{2} 2=\log _{2} 6$. 
f. Find the results for:

$\log _{2} 3+\log _{2} 4 ; \log _{2} 3+\log _{2} 5 ; \log _{2} 3+\log _{2} 7 ; \log _{2} 3+\log _{2} 4 ; \log _{2} 5+\log _{2} 9$

g. What will be $\log _{2} m+\log _{2} n$ for any positive values of $m$ and $n$ ?

h. Why should this be true: $\log _{2} 6-1=\log _{2} 3$ ? And what about: $\log _{2} 3-1=$ $\log _{2}\left(\frac{3}{2}\right) ?$

i. Find the results for:

$$
\log _{2} 10-\log _{2} 4 ; \quad \log _{2} 30-\log _{2} 5 ; \quad \log _{2} 14-\log _{2} 7
$$

j. What will be $\log _{2} m-\log _{2} n$ for any positive values of $m$ and $n$ ?

Recall that $2 \times \log _{2} 3=\log _{2} 3+\log _{2} 3$.

Describe the above equation by completing the sentence:

"When the growth factor is 2 , the time needed to grow ...."

Does this make sense: $\log _{2} 625=4 \times \log _{2} 5$ ? If yes, why?

And what about: $\log _{2}\left(\frac{1}{25}\right)=2 \times \log _{2}\left(\frac{1}{5}\right)$ ?

The three equations of questions above can be rewritten as:

$\log _{2} 3^{2}=2 \times \log _{2} 3$, because $9=3^{2}$

$\log _{2} 5^{4}=4 \times \log _{2} 5$, because $625=5^{4}$

$\log _{2}\left(\frac{1}{5}\right)^{2}=2 \times \log _{2}\left(\frac{1}{5}\right)$, because $\frac{1}{25}=\left(\frac{1}{5}\right)^{2}$.

k. What will be $\log _{2} m^{7}$ for any positive value of $m$ ?

l. Find the value of $x$ in the following equations.

$$
\begin{array}{lll}
\log _{2} x=\log _{2} 5+\log _{2} 7 & \log _{3} x+\log _{3} 5=\log _{3} 35 & \log _{5}(2 x)+\log _{5} 4=\log _{5} 64 \\
\log _{3} x-\log _{3} 5=2 & \log _{2}(2 x+1)=1+\log _{2} 3 & \log _{10} x-\log _{10} 5=\log _{10} 2
\end{array}
$$

\section{Evaluation}

The Dutch Realistic Mathematics Education reform movement entails all parts of Concept Rich Mathematics Instruction. It was elaborated quite earlier than the American reform. The basic aim at both reforms is to build a rich concept images. Unfortunately the American scientists take into the consideration only very few European scientists, maybe that is the reason why the experts of Realistic Mathematics Education are not in the list of References.

It seems to us that the Realistic Mathematics Education reform movement goes further in the direction of Mathematics as science than the Austrian version. 


$$
\text { "Engbersen" — 2009/11/2 — 0:13 — page 317 — \#21 }
$$

\section{Summary}

1. Our assumption was that the teaching of mathematics can be influenced in a great manner by the textbooks. We know that in the reality is it not always true. For this reason our analysis of the textbooks has only partial value.

2. Our opinion is that the realization part in concept teaching is very important. To investigate a lot of Hungarian, Austrian and Dutch mathematics lessons and to ask some teachers from all country could give a more realistic picture about our topics.

3. The mediation role of the teacher is very important. It seems to us that from this point of view the Realistic Mathematics Education gives the best model. It gives the most help for the practicing teachers, if they follow the construction presented in it. Similarly in Austrian textbooks there is a lot of help for teachers. The Hungarian version mirrors science mathematics in a strong manner.

4. To value a construction you need to know for which type of schools, and which students the textbooks are devoted. The Austrian textbook is aimed for technical schools, the Hungarian one is for general Grammar Schools, the Dutch one is for different types of secondary schools. What is quite clear: the Hungarian secondary school mathematics teaching is elite oriented, and does not take into consideration the difficulties of average and below average students. We are sure that the other two textbooks give more help for such students.

5. We find as a good idea to compare the linear and exponential growth. Unfortunately it is not in Hungarian elaboration, only in the other two we have mentioned above. The growth - in general the change - is a very important notion for analysis.

6. At the teaching of functions it is important to use for the students the three different representations (enactive, iconic, symbolic). Two basic operations concept realization and concept identification - are especially important for the acquisition of concepts. Both operations should be used on all three representation levels when teaching functions. Unfortunately there are no such special tasks in the investigated textbooks with this aim. Of course if we take the realistic situations as concrete representations, we may find such problems. On the symbolic level there are many problems too. What we miss is the systemic practice part for concept realizations and identifications 
on all three representation levels after the introduction of exponential and logarithmic functions.

\section{References}

[1] H. Freudenthal, Mathematics as an Educational Task, Reidel, Dodrecht, 1973.

[2] P. Hilton, Education in Mathematics and Science Today: The Spread of False Dichotomies, Proceedings of 3rd ICME, Karlsruhe, 1976.

[3] J. Kosztolányi, I. Kovács, K. Pintér, J. Urbán and I. Vincze, Colourful Mathematics, (Sokszínü Matematika), Mozaik, Szeged, 2003.

[4] M. Kronfellner and W. Peschek, Applied Mathematics 2, (Angewandte Mathematik 2), Öbv and hpt Verlags GMBH, Wien, 1999.

[5] Meir Ben-Hur, Concept-Rich Mathematics Instruction, Association for Supervision and Curriculum Development, Alexandria, Virginia USA, 2005.

[6] J. Piaget, From science of education and the psychology of the child, in: The essential Piaget: An interpretative reference and guide, (H. E. Gruber and J. J. Vonéche, eds.), Northvale, NJ, and London: Jason Aronson, 1995.a, 96.

[7] J. Piaget, Judgment and reasoning in the child (originally published in 1924), in: The essential Piaget: An interpretative reference and guide, (H. E. Gruber and J. J. Vonéche, eds.), Northvale, NJ, and London: Jason Aronson, 1995.b, 96.

[8] R. Skemp, The Psychology of Learning Mathematics, Lawrence Erlbaum Associates Publishers, London, 1987.

[9] D. Tall and S. Vinner, Concept Image and Concept Definition in Mathematics with particular reference to Limits and Continuity, in: Educational Studies in Mathematics, Vol. 12, 1981, 151-169).

[10] A. Treffers, Three dimension, Reidel, Dodrecht, 1986.

[11] Drs. Jan de Lange Jzn, Exponenten en Logaritmen, Instituut Ontwikkeling Wiskunde Onderwijs, Utrecht, 1979.

ARANKA ENGBERSEN

VIENNA INTERNATIONAL SCHOOL

E-mail: engbersen@hotmail.com

(Received May, 2009) 